\title{
A construction for Hadamard arrays
}

\section{Joan Cooper and Jennifer Wallis}

We give a construction for Hadamard arrays and exhibit the arrays of orders $4 t, t \in\{1,3,5,7, \ldots, 19\}$. This gives seventeen new Hadamard matrices of order less than 4000 .

An Hadomard matrix $H$ of order $h$ has every element +1 or -1 and satisfies $H H^{T}=h I_{h}$, where $I$ is the identity matrix of order $h . h$ is necessarily 1,2 or congruent to zero modulo 4 .

The Hadamard product, *, of two matrices $A=\left(a_{i j}\right)$, and $B=\left(b_{i j}\right)$ which are the same size is given by

$$
A * B=\left(a_{i j} b_{i j}\right) \text {. }
$$

We define an Hadamard array of order $4 n$, based on the indeterminates $A, B, C$ and $D$, to be a $4 n \times 4 n$ array with entries chosen from $A,-A$, $B,-B, C,-C, D$ and $-D$ in such a way that:

(i) in any row there are $n$ entries equal to $A$ or $-A, n$ entries $\pm B, n$ entries $\pm C$ and $n$ entries $\pm D ;$ and similarly for columns;

(ii) the rows are formally orthogonal, in the sense that if $A$, $B, C$ and $D$ are realized as any elements of any commutative ring then the rows of the array are pairwise orthogonal; and similarly for columns.

The Hadamard array of order 4 is 


$$
\left[\begin{array}{rrrr}
A & B & C & D \\
-B & A & -D & C \\
-C & D & A & -B \\
-D & -C & B & A
\end{array}\right]
$$

and is due to Williamson [10].

Suppose $V$ is a finite abelian group with $v$ elements, written in additive notation. A difference set $D$ with parameters $(\nu, k, \lambda)$ is a subset of $V$ with $k$ elements and such that in the totality of all the possible differences of elements from $D$ each non-zero element of $V$ occurs $\lambda$ times.

If $V$ is the set of integers modulo $v$ then $D$ is called a cyclic difference set: these are extensively discussed in Baumert [1].

A circulant matrix $B=\left(b_{i j}\right)$ of order $v$ satisfies $b_{i j}=b_{1, j-i+1}$ $(j-i+1$ reduced modulo $v)$, while $B$ is back-circulant if its elements satisfy $b_{i j}=b_{1, i+j-1}(i+j-1$ reduced modulo $v)$.

Throughout the remainder of this paper $I$ will always mean the identity matrix and $J$ the matrix with every element +1 , where the order, unless specifically stated, is determined by the context. The Kronecker product of two matrices will be denoted by $x$.

Let $S_{1}, S_{2}, \ldots, S_{n}$ be subsets of a finite abelian group $V$, $|v|=v$, containing $k_{1}, k_{2}, \ldots, k_{n}$ elements respectively. Write $T_{i}$ for the totality of all differences between elements of $S_{i}$ (with repetitions), and $T$ for the totality of elements of all the $T_{i}$. If $T$ contains each non-zero element of $V$ a fixed number of times, $\lambda$ say, then the sets $s_{1}, s_{2}, \ldots, s_{n}$ will be called $n-\left\{v ; k_{1}, k_{2}, \ldots, k_{n} ; \lambda\right\}$ supplementary difference sets.

The parameters of $n-\left\{v ; k_{1}, k_{2}, \ldots, k_{n} ; \lambda\right\}$ supplementary difference sets satisfy

$$
\lambda(v-1)=\sum_{i=1}^{n} k_{i}\left(k_{i}-1\right) .
$$


If $k_{1}=k_{2}=\ldots=k_{n}=k$ we will write $n-\{v ; k ; \lambda\}$ to denote the supplementary difference sets and (1) becomes

$$
\lambda(v-1)=n k(k-1) \text {. }
$$

See [7] and [8] for more details.

The incidence matrix $A=\left(a_{i j}\right)$ of a subset $X$ of an abelian group $G$ of order $v$, with elements $g_{1}, g_{2}, g_{3}, \ldots, g_{v}$, is found by choosing

$$
a_{i j}= \begin{cases}1 & \text { if } g_{j}-g_{i} \in X, \\ 0 & \text { otherwise. }\end{cases}
$$

If $A_{1}, A_{2}, \ldots, A_{n}$ are the incidence matrices of $n-\left\{v ; k_{1}, k_{2}, \ldots, k_{n} ; \lambda\right\}$ supplementary difference sets then

$$
\sum_{i=1}^{n} A_{i} A_{i}^{T}=\left(\sum_{i=1}^{n} k_{i}-\lambda\right) I+\lambda J
$$

and the $(1,-1)$ matrices $B_{i}=2 A_{i}-J$ satisfy

$$
\sum_{i=1}^{n} B_{i} B_{i}^{T}=\left(4 \sum_{i=1}^{n} k_{i}-4 \lambda\right) I+\left(n v-4 \sum_{i=1}^{n} k_{i}+4 \lambda\right) J .
$$

We define the matrix $R=\left(r_{i j}\right)$ of order $v$ on $G$ by

$$
r_{i j}= \begin{cases}1 & \text { if } g_{i}+g_{j}=0, \\ 0 & \text { otherwise. }\end{cases}
$$

For example, if $G$ is the integers modulo $n$ with the usual ordering,

$$
r_{i, n-i}=1, r_{i j}=0 \text { otherwise. }
$$

\section{The construction}

THEOREM 1. Suppose there exist four $(0,1,-1)$ matrices $X_{1}, X_{2}$, $X_{3}, x_{4}$ of order $n$ which satisfy

(i) $x_{i} * x_{j}=0, i \neq j, i, j=1,2,3,4$, 
(ii) $\sum_{i=1}^{4} x_{i} x_{i}^{T}=n I_{n}$.

Suppose $x_{i}$ is the number of positive elements in each row and colum of $x_{i}$ and $y_{i}$ is the number of negative elements in each row and colum of $x_{i}$. Then

(a) $\sum_{i=1}^{4}\left(x_{i}+y_{i}\right)=n$,

(b) $\sum_{i=1}^{4}\left(x_{i}-y_{i}\right)^{2}=n$.

Proof. (a) follows immediately from (ii). To prove (b) we consider the four $(1,-1)$ matrices

$$
\begin{aligned}
& y_{1}=-x_{1}+x_{2}+x_{3}+x_{4}, \\
& y_{2}=x_{1}-x_{2}+x_{3}+x_{4}, \\
& y_{3}=x_{1}+x_{2}-x_{3}+x_{4}, \\
& y_{4}=x_{1}+x_{2}+x_{3}-x_{4} .
\end{aligned}
$$

From [7] we know that $4-\left\{n ; k_{1}, k_{2}, k_{3}, k_{4} ; \sum_{i=1}^{4} k_{i}-n\right\}$ supplementary difference sets may be used to form an Hadamard matrix of order $4 n$. Now

$$
\sum_{i=1}^{4} Y_{i} Y_{i}^{T}=4 n I_{n}
$$

so $z_{i}=\frac{1}{2}\left(Y_{i}+J\right), i=1,2,3,4$ are the incidence matrices (or permutations of them) of

$$
4-\left\{n ; y_{1}+x_{2}+x_{3}+x_{4}, x_{1}+y_{2}+x_{3}+x_{4}, x_{1}+x_{2}+y_{3}+x_{4}, x_{1}+x_{2}+x_{3}+y_{4} ; 2 \sum_{i=1}^{4} x_{i}\right\}
$$

supplementary difference sets. Using (1) we have

$$
2 \sum_{i=1}^{4} x_{i}(n-1)=\sum_{i=1}^{4}\left(x_{1}+x_{2}+x_{3}+x_{4}+y_{i}-x_{i}\right)\left(x_{1}+x_{2}+x_{3}+x_{4}+y_{i}-x_{i}-1\right) \text {, }
$$


or writing $x_{1}+x_{2}+x_{3}+x_{4}=w, t=y_{1}+y_{2}+y_{3}+y_{4}, n=w+t$,

$$
\begin{aligned}
2 w(n-1) & =\sum_{i=1}^{4}\left(x+y_{i}-x_{i}\right)\left(w+y_{i}-x_{i}-1\right) \\
& =4 w^{2}+2 w \sum_{i=1}^{4}\left(y_{i}-x_{i}\right)+\sum_{i=1}^{4}\left(y_{i}-x_{i}\right)^{2}-\sum_{i=1}^{4}\left(y_{i}-x_{i}\right)-4 w \\
& =4 w^{2}+2 w(t-w)+\sum_{i=1}^{4}\left(y_{i}-x_{i}\right)^{2}-(t-w)-4 w .
\end{aligned}
$$

So

$$
\sum_{i=1}^{4}\left(y_{i}-x_{i}\right)^{2}=n,
$$

as required.

THEOREM 2. Suppose there exist four $(0,1,-1)$ circulant matrices $x_{1}, x_{2}, x_{3}, x_{4}$ of order $n$ satisfying the conditions of the above theorem. Then there exists an Hadamard array of order $4 n$.

Proof. Consider the following matrices, where $A, B, C, D$ are indeterminates which commute in pairs

$$
\begin{aligned}
& y_{1}=X_{1} \times A+X_{2} \times B+X_{3} \times C+X_{4} \times D, \\
& y_{2}=X_{1} \times-B+X_{2} \times A+X_{3} \times D+X_{4} \times-C, \\
& Y_{3}=X_{1} \times-C+X_{2} \times-D+X_{3} \times A+X_{4} \times B, \\
& y_{4}=X_{1} \times-D+X_{2} \times C+X_{3} \times-B+X_{4} \times-A,
\end{aligned}
$$

and

$$
H=\left[\begin{array}{cccc}
y_{1} & y_{2} R & Y_{3} R & Y_{4}^{R} \\
-Y_{2} R & y_{1} & -y_{4}^{T} R & Y_{3}^{T} R \\
-y_{3} R & Y_{4}^{T} R & Y_{1} & -Y_{2}^{T} R \\
-Y_{4} R & -Y_{3} R & Y_{2}^{T} R & Y_{1}^{T}
\end{array}\right],
$$

where $R$ is the Goethals-Seidel matrix (see $[3,6]$ ).

Now clearly $B$ is of order $4 n$. Since each indeterminate is 
associated with $X_{1}, X_{2}, X_{3}$ and $X_{4}$ in each row and column, and (a) of Theorem 1 holds, each indeterminate occurs exactly $n$ times in each row and column. It may be verified that

$$
H H^{T}=I_{4} \times \sum_{i=1}^{4} Y_{i} Y_{i}^{T}
$$

It remains to show that

$$
\sum_{i=1}^{4} Y_{i} Y_{i}^{T}=n I_{n} \times\left(A A^{T}+B B^{T}+C C^{T}+D D^{T}\right)
$$

but this is clearly true since $\sum_{i=1}^{4} X_{i} X_{i}^{T}=n I_{n}$.

There is an equivalent enunciation for both Theorems 2 and 3 when $X_{1}, X_{2}, X_{3}, X_{4}$ are matrices defined on subsets of abelian groups.

THEOREM 3. Suppose there exist four circulant $(0,1,-1)$ matrices $x_{1}, X_{2}, X_{3}, x_{4}$ of order $n$ which satisfy

(i) $x_{i} * x_{j}=0, i \neq j, i, j=1,2,3,4$,

(ii) $\sum_{i=1}^{4} X_{i} X_{i}^{T}=n I_{n}$.

Further suppose there exist four $(1,-1)$ matrices $A, B, C, D$ of order $m$ which pairwise satisfy $M N^{T}=N M^{T}$ and for which

$$
A A^{T}+B B^{T}+C C^{T}+D D^{T}=4 m I_{m}
$$

Then there exists an Hadamard matrix of order $4 m n$.

Proof. This follows by replacing the indeterminates $A, B, C, D$ of the previous theorem by the matrices $A, B, C, D$.

COROLLARY 4. There exists an Hadamard array of order $4 t$ for $t \in\{x: x$ is an odd integer, $1 \leq t \leq 19\}$.

Proof. The matrices $x_{1}, X_{2}, X_{3}, x_{4}$ for $t=7,9,11$ may be found in [6]. These matrices were found by Welch for $t=5$, (unpublished result) but we give it here for completeness. 
In each case we give a set which may be used to determine the first row of $x_{1}, x_{2}, x_{3}, x_{4}$. This is possible because the $x_{i}$ are circulant. If $\pm i$ is in the set for $X_{j}$ then the $i$-th element of the first row of $X_{j}$ is \pm 1 , all the other elements are zero. Use the sets from the following table:

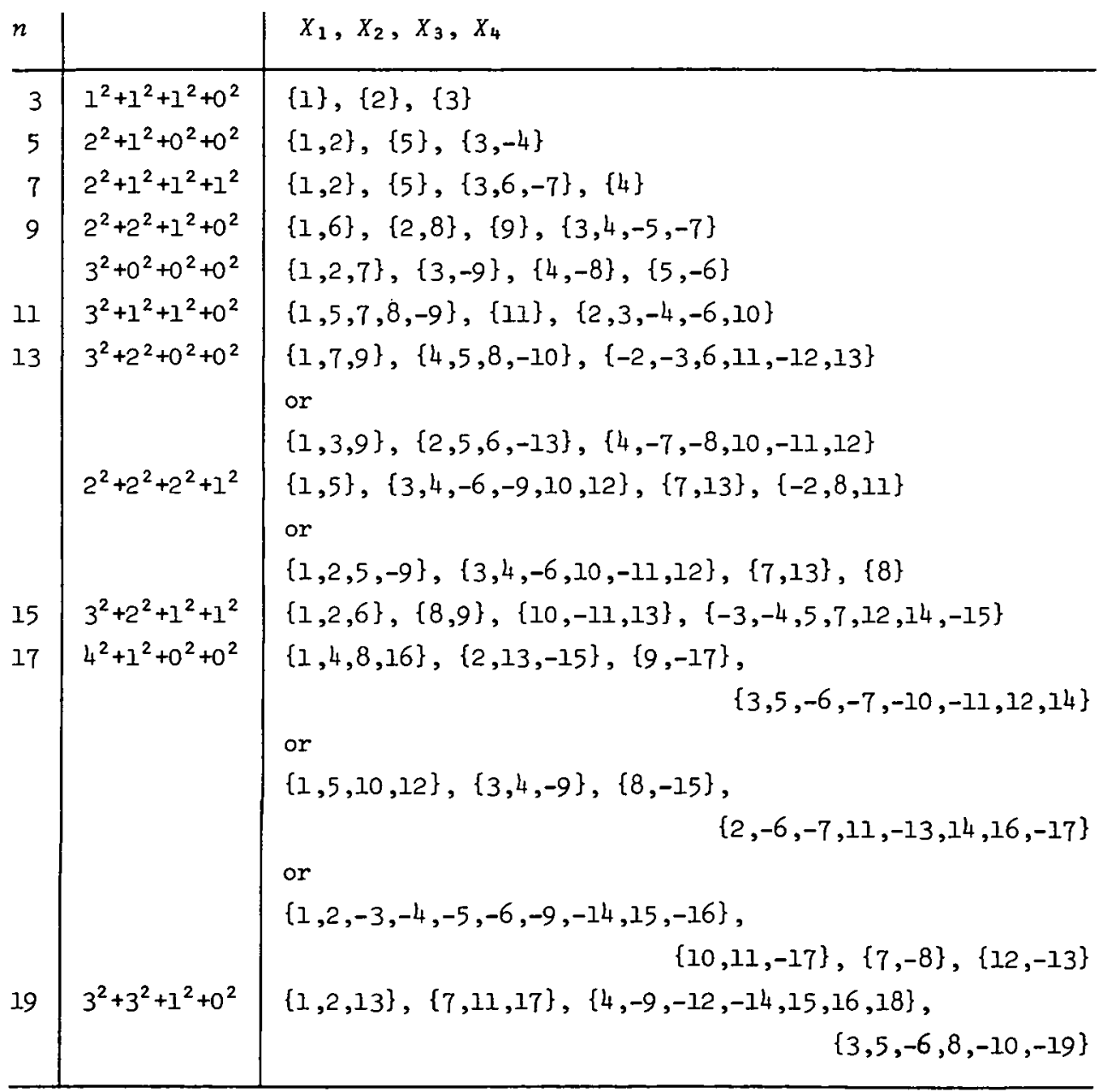

The matrices $x_{1}, x_{2}, x_{3}, x_{4}$ for $n=13=3^{2}+2^{2}+0^{2}+0^{2}$ were found by listing the multiplicative cyclic group of order 12 generated by 2 to form the subgroup $c_{0}=\left\{2^{4 j}: j=0,1,2\right\}$ of order 3 and its 
cosets $C_{i}=\left\{2^{4 j+i}: j=0,1,2\right\}, i=1,2,3$. Then the first rows of $x_{1}, x_{2}, x_{3}, x_{4}$ may be obtained by using the sets

$$
c_{0} \cup\left(-c_{1}\right), c_{3} \cup\{-13\}, c_{2}, \varnothing
$$

or

$$
c_{2} \cup\left(-c_{3}\right), c_{1} \cup\{-13\}, c_{0}, \emptyset
$$

where $-C_{i}=\left\{-i: i \in C_{i}\right\}$, and the $x_{j}$ are formed as described in the proof of Corollary 4 .

For $n=19=3^{2}+3^{2}+1^{2}+0^{2}$ the multiplicative cyclic group of order 18 generated by 2 was used to form the subgroup $c_{0}=\left\{2^{6 j}: j=0,1,2\right\}$ of order 3 and its cosets $c_{i}=\left\{2^{6 j+i}: j=0,1,2\right\}, i=1, \ldots, 5$. Then $x_{1}, x_{2}, x_{3}, x_{4}$ were found, as above, by using the sets

$$
C_{1}, C_{3}, C_{2} \cup\left\{-C_{1}\right\},\{0\} \cup C_{4} \cup\left\{-C_{5}\right\}
$$

Matrices $A, B, C$ and $D$ satisfying the conditions of Theorem 3 have previously been used to construct Hadamard matrices of orders $4 m[10]$, $12 m$ [2], $20 m$ (unpublished result of Welch, communicated to the authors by Baumert), $28 \mathrm{~m}, 36 \mathrm{~m}, 44 \mathrm{~m}$ [6]. They are known to exist when $m$ is a member of the set

$$
M=\{3,5,7, \ldots, 29,37,43\},
$$

[4], and when $2 m-1$ is a prime power congruent to 1 modulo 4 [5, 9].

COROLLARY 5. There exist Hadamard matrices of orders $52 \mathrm{~m}, 60 \mathrm{~m}$, $68 m, 76 m$ whenever $m \in M$.

COROLLARY 6. There exist Hadomard matrices of orders $26(q+1)$, $30(q+1), 34(q+1), 38(q+1)$ whenever $q$ is a prime power congruent to 1 moduzo 4 .

This gives the following new Hadamard matrices of order < 4000: $988,1196,1444,1508,1564,1612,1900,1972,2108$, $2356,2516,2788,2924,3116,3128,3172,3876$. 


\section{References}

[1] Leonard D. Baumert, Cyclic difference sets (Lecture Notes in Mathematics, 182. Springer-Verlag, Berlin, Heidelberg, New York, 1971).

[2] k.D. Baumert and Marshall Hall, Jr, "A new construction for Hadamard matrices", Bulz. Amer. Math. Soc. 71 (1965), 169-170.

[3] J.M. Goethals and J.J. Seidel, "A skew Hadamard matrix of order 36 ", J. Austral. Math. Soc. 11 (1970), 343-344.

[4] Marshall Hall, Jr, Combinatorial theory (Blaisdell Publishing Co. [Ginn \& Co.], Waltham, Massachusetts; Toronto; London; 1967).

[5] Richard J. Turyn, "An infinite class of Williamson matrices", $J$. Combinatorial Theory 12 (1972), 319-321.

[6] Jennifer Wallis, "Hadamard matrices of order $28 \mathrm{~m}, 36 \mathrm{~m}$ and $44 \mathrm{~m}$ ", J. Combinatorial Theory (to appear).

[7] Jennifer Wallis, "On supplementary difference sets", Aequationes Math. (to appear).

[8] Jennifer Wallis, "A note on BIBDs", J. Austral. Math. Soc. (to appear).

[9] Albert Leon Whiteman, "An infinite family of skew Hadamard matrices", (to appear).

[10] John Williamson, "Hadamard's determinant theorem and the sum of few squares", Duke J. Math. 11 (1944), 65-81.

Department of Mathematics, University of Newcastle, New South Wales. 